\title{
Introduction to the Personal Data: Analytics and Management
}

\author{
Joo Hee Oh \\ WMG \\ University of Warwick \\ joo.oh@warwick.ac.uk
}

\author{
Philip Davies \\ Henley Business School \\ University of Reading \\ philip.davies@henley.ac.uk
}

\author{
Glenn Parry \\ Surrey Business School \\ University of Surrey \\ G.parry@surrey.ac.uk
}

For the first time, HICSS-54 (January 5-8, 2021, Kauai) features a minitrack on Personal Data as part of the Decision Analytics and Service Science track. The minitrack invited researchers and practitioners in the emerging field of personal data analytics and service science to address how the introduction of consumer decision-making into the data-analytic mix could impact ecosystems and business models. To unlock the most promising opportunities and release the potential of personal data, personal data ecosystems require design and management that ensures personal data remains available for analysis, whilst protecting the rights of the individual. Knowing the value and utility of personal data can empower intelligent consumerism within social networks and provide collective insights at the society level, providing businesses with opportunities to achieve substantial competitive advantage. Research focuses on various domains related to next generation of business intelligence, analytics and challenges with personal data privacy, value and regulation. Papers include theory development, empirical studies, case studies and other high-quality research manuscripts. In 2021 the minitrack features three papers. Contributions cover personal data value, privacy and decision support models for data ecosystems that will stimulate further discussion and exploration of the key phenomena within this domain.

In the first paper, "Healthy surveillance: Designing a concept for privacy-preserving mask recognition AI in the age of pandemics" by Niklas K"uhl, Dominik Martin, Clemens Wolff, and Melanie Volkamer (all from Karlsruhe Institute of Technology, Germany) research shows that a privacy-preserving mask recognition artifact demonstrated high detection performance, between $95 \%$ and $99 \%$, with different options for implementation. The authors explore the benefits of privacy-preserving technology as well as deep-learning based on artificial intelligence (AI) based on the trade-off between the level of privacy preservation and Artificial Intelligence performance, i.e. the "price of privacy".

The second paper is titled "Circular Insurance: customer-centric, data-driven services for the Circular Economy" and authored by Phil Godsiff and Zena Wood (both University of Exeter Business School). In their research paper, the authors build and propose a new finance insurance ecosystem, Circular Insurance. Circular insurance is a customer centric, data-driven approach for business models using the exemplary field of insurance. The resulting artifact reveals a continuous data collection, analysis and interpretation process via a Risk Management Service Platform (RMSP). The artifact uses Personal Data Stores (PDS) and a Data Analytics Engine (DAE) to enable the desired outcome. This framework gives service providers a tool, based on innovative digital technologies, that encourages and rewards behaviours that embrace and enhance circular economy (CE) design principles, leading to competitive advantages.

In the third paper, "Data are in the Eye of the Beholder: Co-creating the Value of Personal Data" and authored by Marta Stelmaszak (London School of Economics) and one of the co-chairs of this mini-track, Glenn Parry (Surrey Business School) conceptualizes how the value of personal data emerges as multi-faceted, dynamic, and co-created by stakeholders based on different epistemological stances. The author's main argument is that the value of personal data is not static, defined or fixed, but is an outcome of negotiations and trade-offs among multiple stakeholders whose perceptions of value might not always be congruent.

Together, this minitrack creates a community of scholars who present findings and create future synergies around the emerging phenomenon of personal analytics and debate the future of personal data based services. 